\title{
A Simple Theory for Vibration of MRI Gradient Coils
}

\author{
D. Tomasi ${ }^{1}$ and T. Ernst ${ }^{2}$ \\ ${ }^{1}$ Medical Department, Brookhaven National Laboratory, Upton, NY, 11973 and \\ ${ }^{2}$ Department of Medicine, Hawai University, Honolulu, HI, 96813
}

Received on 28 August, 2005; accepted on 28 November, 2005

\begin{abstract}
Vibrations of a string can provide a model for vibrations of MRI coil assemblies. The string model for the vibrations of MRI coils is presented, and compared with experimental results. Gradient coils exhibit resonant modes because of the finite coil length, $l$, and the elasticity of materials that comprise the assembly. The resonance frequencies depend on $l$ and the Young modulus, as well as on the current distribution. Under longitudinal gradient pulses, anti-symmetrical modes of surface vibration are produced due to the symmetry of current distribution. In contrast, transverse gradient pulses produce coil bending that results in symmetrical vibration modes of the coil axis. The viscosity of the assembly materials controls the width of the vibrational resonance modes. Experimental results are in agreement with this simple string model.
\end{abstract}

Keywords: MRI; MRI coils

\section{INTRODUCTION}

The high-intensity acoustic noise produced by fast MRI techniques, such as echo-planar imaging (EPI), has been a concern for patient safety and comfort.The sound pressure level (SPL) of acoustic noise in MRI systems can exceed $100 \mathrm{~dB}$, and may cause anxiety and discomfort in patients [1]. Acoustic noise can also obscure functional magnetic resonance imaging (fMRI) studies. For instance, loud acoustic noise can produce spurious areas of activation in the auditory cortex [2-3] or alter the BOLD signal in motor and visual cortices [4-5]. Acoustic noise is a consequence of the vibrations produced in the coil assembly by the Lorentz interaction between the static magnetic field, $B_{0}$, and the time-dependent currents in gradient wires. The SPL of acoustic noise in MRI scanners is commonly reduced by the use of acoustic absorbing materials and ear protection (ear plugs and headphones). In addition, the SPL can be reduced by the development of "silent" MRI-pulse sequences [6-9] or the design of "quiet" gradient coils [10-13]. Several experimental studies [14-17] have demonstrated a clear increase in SPL of acoustic noise for high-field systems [14], and the existence of acoustic resonances [15]. However, the vibrational motion of gradient assemblies, where the acoustic noise originates, has not been studied extensively. Therefore, we developed a simple model, based on the vibrations of an elastic string, to understand the vibration motion of gradient assemblies. Additionally, we measured the vibration motion of our gradient coil to test the model.

\section{THEORY}

\section{Longitudinal gradients}

Since MRI gradient coils are operated in magnetic fields, a Lorentz force acts on the coil wires during gradient current pulses. We assume that the main magnetic field $B_{0}$ is along the $z$-axis. For a current density, $j(\phi, z, t)$, on a cylindrical surface of radius $a$, the magnetic force element, $d \mathbf{F}$, is a radial vector caused by the $\phi$-component of the current density, $j_{\phi}(\phi, z, t)$ :

$$
d F(\phi, z, t)=a B_{0} j_{\phi}(\phi, z, t) d \phi d z
$$

For longitudinal gradient coils, $j$ does not depend on $\phi$, and as a consequence, the magnetic force $d \mathbf{F}(z, t)$ is axially symmetric with respect to $B_{0}$. Because the current distribution is anti-symmetrical along $z$ in longitudinal coils, the magnetic force is compressing one half of the coil, while expanding the other half. This results in radial waves that travel along the coil assembly. The flexible string is a simple and wellunderstood system that can be used to model vibrations in longitudinal gradient coils. In this model, the amplitude of radial displacements of coil assembly, $\zeta$, due to a driving force per unit area, $f\left(z_{0}, t\right)$, exerted at axial position $z=z_{0}$, is given by

$$
\rho \frac{\partial^{2} \zeta(z, t)}{\partial t^{2}}+\eta \frac{\partial \zeta(z, t)}{\partial t}-E \frac{\partial^{2} \zeta(z, t)}{\partial z^{2}}=f\left(z_{0}, t\right) \delta\left(z-z_{0}\right)
$$

where, $\rho$, and $E$, are the mass and Young's modulus per unit area of the material used to hold the wires. These elastic properties of the coil assembly determine the velocity of traveling waves, $c=\sqrt{E / \rho}$. The resistive force per unit area, opposing the assembly's motion, is due to the viscosity of the medium, $\eta$, which gains the energy lost by the "string". A fraction of this energy is dissipated into heat, and the remaining fraction is converted into sound waves in air. For a simple harmonic current flowing in the coil with frequency $\omega / 2 \pi$, and considering an effective frictional resistance per unit area, $2 k=\eta / \rho$, the equation of motion can be written as:

$$
\begin{gathered}
\frac{\partial^{2} \zeta(z, t)}{\partial t^{2}}+2 k \frac{\partial \zeta(z, t)}{\partial t}-c^{2} \frac{\partial^{2} \zeta(z, t)}{\partial z^{2}}= \\
\frac{B_{0} j\left(z_{0}\right) \delta\left(z-z_{0}\right)}{\rho} \exp (-i \omega t),
\end{gathered}
$$

The possible harmonic vibration modes of the "string" are limited, because the coil length, $l$, is finite. For instance, if the 
coil is fixed at both ends we require for $z=0$ and $z=l$, and only a discrete set of vibration frequencies is allowed:

$$
\sin \left(\frac{\omega l}{c}\right)=0 \quad\left(\omega= \pm \frac{\pi c}{l}, \pm \frac{2 \pi c}{l}, \cdots, \pm \frac{n \pi c}{l}\right)
$$

In general, the motion of the string is a combination of all different modes, and can be represented by the Fourier series:

$$
\zeta(z, t)=\sum_{n=1}^{\infty} A_{n} \sin \left(\frac{n \pi z}{l}\right) \exp (-i \omega t)
$$

The Fourier coefficients, $A_{n}$, can be obtained by substituting Eq. (5) into Eq. (3), multiplying both sides by $\sin \left(\frac{n \pi z}{l}\right)$, and integrating over $z$ from 0 to $l$ :

$$
A_{n}=\frac{4 \pi a B_{0} j\left(z_{0}\right) d z_{0}}{M\left(\omega+i k-\omega_{n}\right)\left(\omega+i k+\omega_{n}\right)} \sin \left(\frac{n \pi z_{0}}{l}\right)
$$

where $M$ is the mass of the assembly. To find the response of the string to a simple harmonic driving force distributed along the coil, we integrate Eq. (5) over $z_{0}$ from 0 to $l$

$$
\zeta(z, t)=\frac{4 \pi a B_{0}}{M} \sum_{n-1}^{\infty} \frac{\mathcal{F}_{n} \sin \left(\frac{n \pi z}{l}\right) \exp (-i \omega t)}{\left(\omega+i k-\omega_{n}\right)\left(\omega+i k+\omega_{n}\right)}
$$

where the frequencies of normal modes of vibration, $\omega_{n} / 2 \pi$, depend on elastic properties of the coil assembly, and are given by

$$
\omega_{n}^{2}=\left(\frac{n \pi c}{l}\right)^{2}-k^{2}
$$

It is apparent that friction dampens free vibrations and slightly changes the resonance frequencies. The current-form factor of order $n, \mathcal{F}_{n}$, in Eq. (7) is given by,

$$
\mathcal{F}_{n}=\int_{0}^{l} j\left(z_{0}\right) \sin \left(\frac{n \pi z_{0}}{l}\right) d z_{0}
$$

and represents the overlap between the current distribution and a given spatial harmonic of order $n$. For standard longitudinal gradient coils, $\mathcal{F}_{n}=0$ for odd values of $n$, because $j(z)$ is anti-symmetric with respect to $z=l / 2$. The response of the "string" to a current-impulse, $j(z) \delta(t)$, in the gradient coil can be obtained by using the Fourier expansion of delta function

$$
\delta(t)=\frac{1}{2 \pi} \int_{-\infty}^{\infty} \exp (-i \omega t) d \omega
$$

By integrating Eq. (7) over $\omega$, the dampened vibrations of the coil surface following a single gradient impulse can be written as

$$
\zeta(z, t)=\frac{2 \pi a B_{0}}{M} \exp (-k t) \sum_{n-1}^{\infty} \frac{\mathcal{F}_{n} \sin \left(\frac{n \pi z}{l}\right) \sin \left(\omega_{n} t\right)}{\omega_{n}}
$$

It must be noted that Eq. (11) assumes that the viscosity of the coil assembly is not dependent on the vibration frequency. Transverse gradients
The string model can also be applied to the study of vibrations produced by transverse gradients. However, because the current density, $j(z, \phi)=j(z, 0) \cos (\phi)$, is not axially symmetric for transverse gradient coils, transverse gradient pulses bend the coil. Furthermore, in contrast to the longitudinal case, $\mathcal{F}_{n}=0$ for even values of $n$, because the current density is symmetric with respect to $z=l / 2$ in transverse gradients. Due to the $\cos (\phi)$-dependence of current density in the $x$-gradient coil, the coil center vibrates along $x$ with frequency $\omega / 2 \pi$ due to the magnetic torque, which bends the coil. In this case, the departure from equilibrium of the coil axis, $X(z, t)$, can also be modeled by the flexible string model. For instance, bending of the coil due to currents in the $x$-transverse coils produces $x$-transverse waves that travel along $z$ with velocity $C$. Note that we will use upper case characters to distinguish vibrations of the coil axis from surface vibrations of the coil assembly.

In analogy to the longitudinal case [Eq. (3)], the equation of motion as a result of a harmonic current flowing at $z=z_{0}$ with frequency $\omega / 2 \pi$ is:

$$
\begin{gathered}
\frac{\partial^{2} X(z, t)}{\partial t^{2}}+2 K \frac{\partial X(z, t)}{\partial t}-C^{2} \frac{\partial^{2} X(z, t)}{\partial z^{2}}= \\
\frac{B_{0} I\left(z_{0}\right) \delta\left(z-z_{0}\right)}{a \rho \pi} \exp (-i \omega t),
\end{gathered}
$$

where $I\left(z_{0}\right)$ is the current per unit length flowing in the coil at axial position $z=z_{0}$ :

$$
\begin{gathered}
I\left(z_{0}\right)=2 a \int_{-\pi / 2}^{\pi / 2} j\left(z_{0}, \phi\right) d \phi= \\
2 a \int_{-\pi / 2}^{\pi / 2} j\left(z_{0}, 0\right) \cos (\phi) d \phi=4 a j\left(z_{0}, 0\right)
\end{gathered}
$$

The response of the string to a simple harmonic driving force distributed along the coil is given by

$$
X(z, t)=\frac{16 a B_{0}}{M} \sum_{n-1}^{\infty} \frac{\mathcal{F}_{n} \sin \left(\frac{n \pi z}{l}\right) \exp (-i \omega t)}{\left(\omega+i K-\Omega_{n}\right)\left(\omega+i K+\Omega_{n}\right)}
$$

with

$$
\Omega_{n}^{2}=\left(\frac{n \pi C}{l}\right)^{2}-K^{2}
$$

Damped vibrations of the coil axis following a currentimpulse in the transverse gradient coil are given by

$$
X(z, t)=\frac{16 a B_{0}}{M} \exp (-K t) \sum_{n-1}^{\infty} \frac{\mathcal{F}_{n} \sin \left(\frac{n \pi z}{l}\right) \sin \left(\Omega_{n} t\right)}{\Omega_{n}}
$$

As an example, Fig. 1(a) shows the amplitude of coil assembly vibrations as a function of frequency, when sine currents drive the longitudinal and transverse gradient coils (Figs. 1b 
and c). These calculations were performed using Eqs. (7) and (14), and assuming $l=1 \mathrm{~m}, c=C=1600 \mathrm{~m} / \mathrm{s}, a=0.35 \mathrm{~m}$, $k=K=100 \mathrm{~Hz}$, and using the parameters listed in Fig. 1c. For the transverse coil $z=0.5 \mathrm{~m}$, while for the longitudinal coil $\mathrm{z}=0.45 \mathrm{~m}$. Figs. $1 \mathrm{~b}$ and $1 \mathrm{c}$ show one octant of the transverse, and half of the longitudinal coil layout. These unshielded gradient coils were designed by using the fastsimulated annealing method [18] to produce 32 and $44 \mathrm{mT} / \mathrm{m}$, when driven by $300 \mathrm{~A}$ currents, respectively. The amplitude of vibrations peaks at discrete frequencies, and does not exceed $50 \mu \mathrm{m}$ (Fig. 1c). It is obvious that longitudinal and transverse gradient coils produce different normal modes of vibration. Because the amplitude of sound in air is proportional to the amplitude of the coil vibrations [17], the linear increase of the gradient coil forces and vibrations with $B_{0}$ cause a logarithmic increase in SPL levels (in $\mathrm{dB}$ ), in agreement with experimental results [14]. In addition, Eq. (7) and (14) demonstrate that the amplitude of vibrations increases with coil radius and decreases with coil mass. The relative amplitude of each normal mode depends on the current distribution.

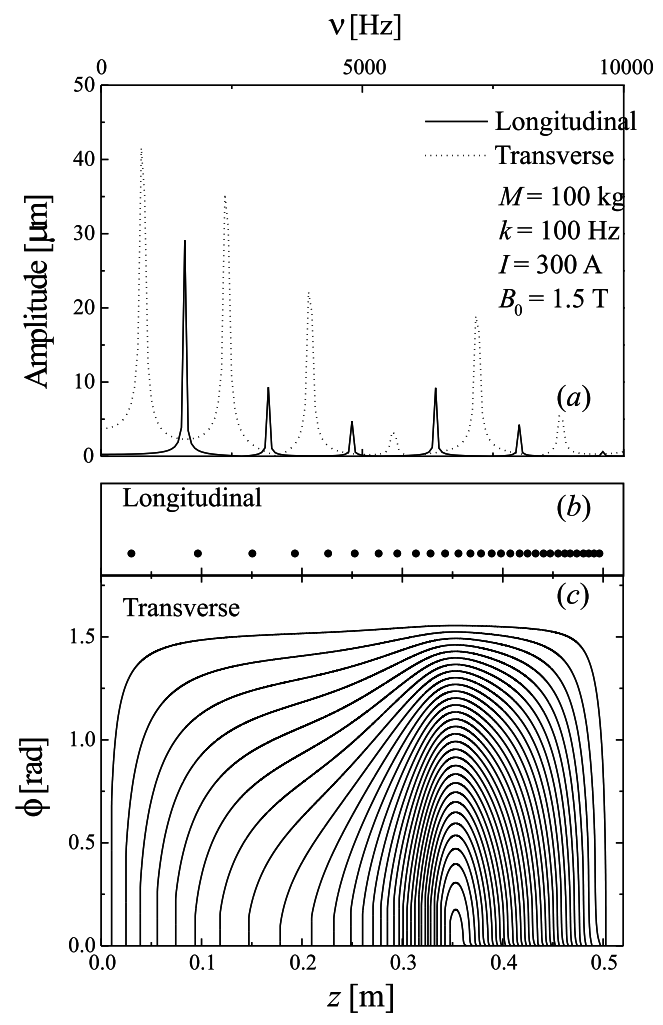

FIG. 1: (a) Theoretical vibration amplitudes for harmonic driving currents as a function of frequency for longitudinal and transverse gradients. (b) Longitudinal coil layout. (c) Transverse coil layout (one octant). $l=1 \mathrm{~m}, a=0.35 \mathrm{~m}, c=C=1600 \mathrm{~m} / \mathrm{s}$, and $k=K=$ $100 \mathrm{~Hz}$.

\section{METHODS}

To measure the mechanical vibrations of a gradient coil, three contact microphones were placed at different positions on the inner surface of a whole-body shielded SONATASiemens gradient set. The mass, length and inner radius of this gradient assembly are $775 \mathrm{~kg}, 1.245 \mathrm{~m}$, and $0.3415 \mathrm{~m}$, respectively. Three K2217 Siemens Cascade Gradient Power Amplifier (2000 V \& 500 A) are used to produce gradient pulses with $44 \mathrm{mT} / \mathrm{m}$ peak amplitude and $0.25 \mathrm{~ms}$ rise-time. This gradient system was operated in a magnetic field of $B_{0}=$ $4 \mathrm{~T}$ and was driven by a Varian INOVA console.

The contact microphones (PZT) consisted of piezoelectric transducers (Radio Shack, 273-073A), which are ideal for use at high magnetic fields because they are non-magnetic. To estimate the frequency response of PZT microphones, we used a waveform generator to produce PZT-vibrations and power absorption of PZT microphones was measured as a function of frequency. These piezoelectric transducers have a non-uniform frequency response, being more sensitive to vibrations in the range of 500-3500 Hz. The microphones were glued on the inner wall of the gradient coil at positions: $(a, 0,0),(0, a, 0)$, and $(0,-a, l / 2)$ and were labeled $x$ PZT, $y$-PZT and $z$-PZT $(a=0.3415 \mathrm{~m})$. The output voltages of the microphones were digitized with a digital oscilloscope (LECROY 9354TM, 50ms trace, 500kHz-sampling rate, 16bits dynamic range, high-impedance) and data analysis (FFT) was done in Microcal Origin (Microcal Software inc.) on a Personal Computer.

To measure the impulse response of the coil assembly, brief rectangular gradient pulses with 250A current amplitude $(22 \mathrm{mT} / \mathrm{m})$ and $500 \mu \mathrm{sec}$-duration were applied, separately for each axis [19]. To evaluate the dynamic behavior of the gradient coil and identify potential higher-order normal modes of vibration, sine- and sinc-shaped gradient pulses were used. Sine pulses of 500 periods, with variable duration in the range 80-1000 ms, were used to sweep the frequency of the driving current in the range $500-5000 \mathrm{~Hz}$. With these pulses, PZT signals demonstrate an approximately $20 \mathrm{~ms}$-transient state, followed by a steady state, and another final $20 \mathrm{~ms}$-transient state (Fig. 5a). The amplitude of vibrations was measured in steady state conditions. Alternatively, sinc-pulses with 300 periods and $50 \mathrm{~ms}$ duration were used to excite all high-order vibration modes by a single waveform, by taking advantage of the flat, 6kHz-bandwidth spectral density of the sinc-pulses. In this case, signal acquisition was triggered at the maximum of the gradient pulse (Fig. 5b). The driving current was 30A for sine-, and $125 \mathrm{~A}$ for sinc-gradient pulses

\section{RESULTS}

\section{Longitudinal impulse-response}

Figure 2 shows damped oscillations of PZT-signals caused by a gradient pulse in the longitudinal gradient coil. The frictional resistance of the coil assembly, $2 k=160 \mathrm{~Hz}$, was obtained from Eq. (11) by fitting a mono-exponential decay to the envelope of the PZT signal. The $x$ - and $y$-PZT signals are smaller than the $z$-PZT signal due to the anti-symmetrical distribution of magnetic force in the longitudinal gradient.

Figure 3 shows the corresponding spectral densities obtained by Fourier transformation of PZT-signals. The peaks 


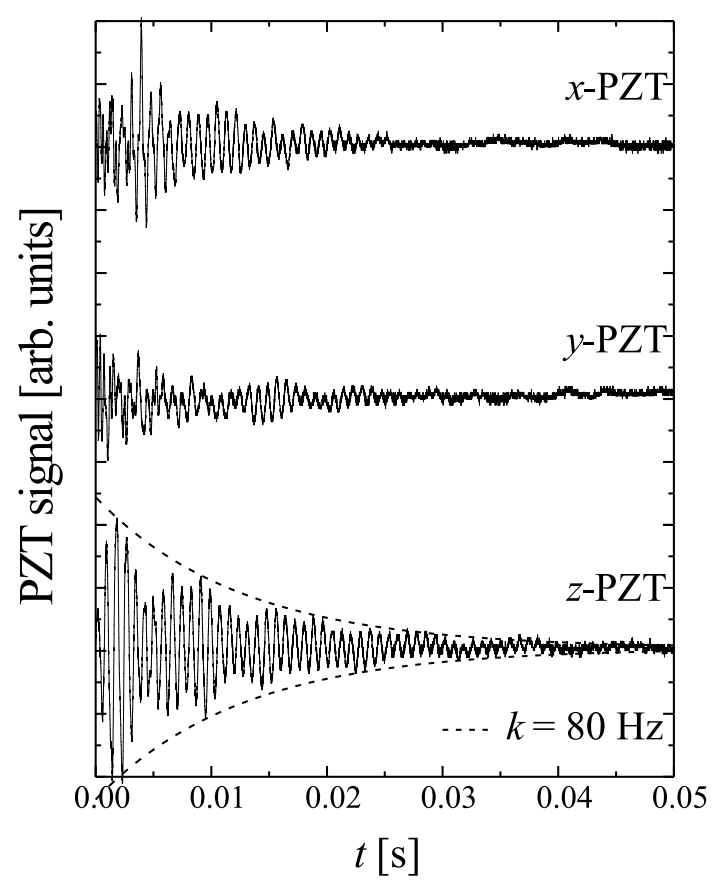

FIG. 2: Experimental vibration of coil assembly caused by a currentimpulse in the $G_{z}$ coil, as a function of time. Gradient amplitude 22 $\mathrm{mT} / \mathrm{m}$, pulse duration $0.5 \mathrm{~ms}$. Dashed lines represent the exponential decay of damped oscillations.

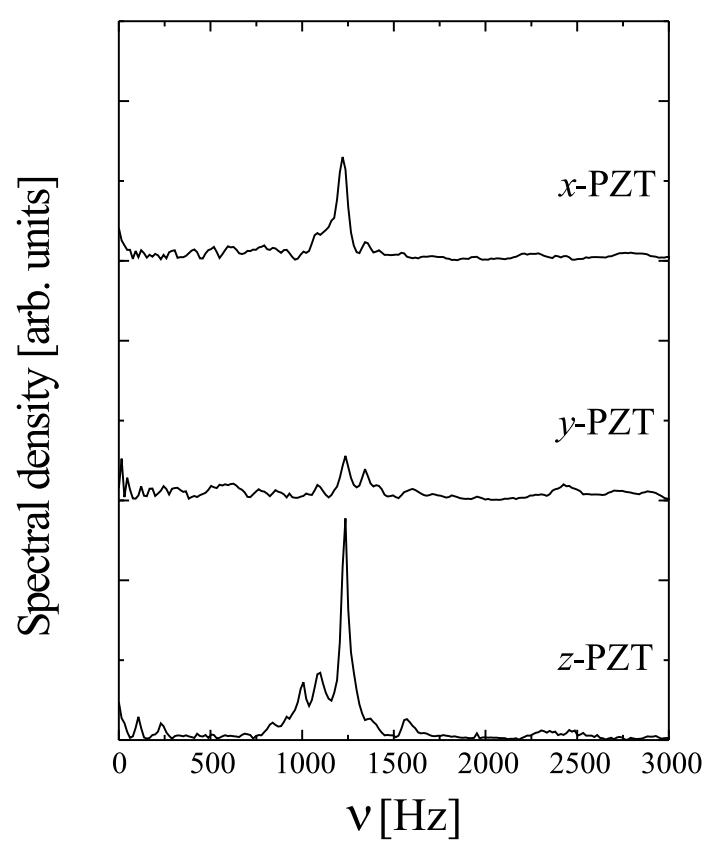

FIG. 3: Fourier amplitudes of PZT signals from FIG.2.

in spectral density represent resonant vibration modes of the coil assembly. The exponential decay of signals in Fig. 2 result in Lorentzian-shaped peaks of $40 \mathrm{~Hz}$ full width half maximum (FWHM), in agreement with Eq. (11).

The z-PZT spectral density has significant amplitude only in the range between $900-1400 \mathrm{~Hz}$, and peaks at 1007,1090 , and $1235 \mathrm{~Hz}$ (Fig. 3, bottom). These resonant modes, which correspond to $n=2$, have different resonance frequencies because of the complex structure of the coil assembly. Higher vibration-modes were not observed in this experiment, probably due to the limited bandwidth $(\sim 2 \mathrm{kHz})$ of the gradient pulses. Using Eq. (8) and $l=1.25 \mathrm{~m}$, the velocity of traveling waves for these resonant modes were calculated to be $c=$ 1260,1360 , and, $1540 \mathrm{~m} / \mathrm{s}$.

\section{Transverse impulse-response}

Figure 4 shows the spectral density of PZT signals following a brief gradient pulse on the $x$-gradient channel. While the resulting $x$-PZT spectrum shows resonance frequencies at 720 and $1220 \mathrm{~Hz}$, the $y$-PZT and z-PZT channels only exhibit the $720 \mathrm{~Hz}$ resonance. The even resonance mode $(1220 \mathrm{~Hz})$ of the $x$-channel suggests that, due to imperfections of coil ends fixation, the $x$-transverse gradient can produce vibrations of the coil assembly's surface at $y=0$ but not at $x=0$ in accord with a current distribution along $\phi$ in this gradient channel.

Our model suggests that the $720 \mathrm{~Hz}$ resonance represents the fundamental mode $(n=1)$ of axis-vibration due to coil bending. The velocity of traveling waves for these axisvibrations was calculated to be $c=1800 \mathrm{~m} / \mathrm{s}$, using Eqs. (8) and (15).

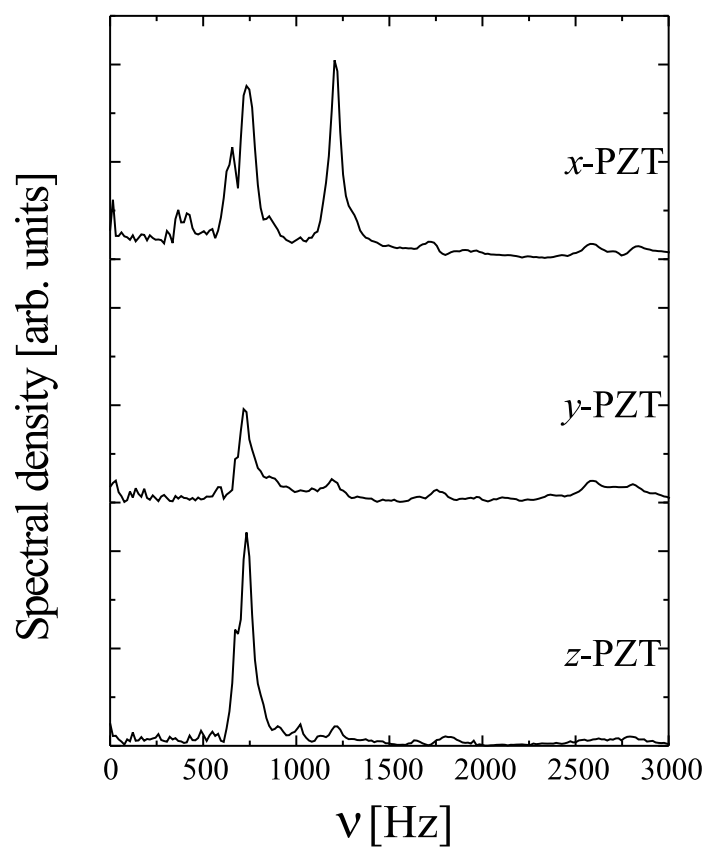

FIG. 4: Fourier amplitudes of coil vibration caused by a currentimpulse in the $G_{x}$ coil. Gradient amplitude $22 \mathrm{mT} / \mathrm{m}$, pulse duration $0.5 \mathrm{~ms}$.

\section{Dynamic-response experiment}

Figure $5 \mathrm{c}$ shows the spectral density of the $x$-PZT-signal when the $G_{x}$-coil is driven by sine-shaped (solid line) and sinc-shaped (dotted line) currents. Both methods show the same set of resonant modes.

When the gradient coils $G_{x}$, and $G_{y}$ apply the driving force, the amplitude of vibrations peaks at similar frequencies, due to their similar current distribution. In contrast, the 


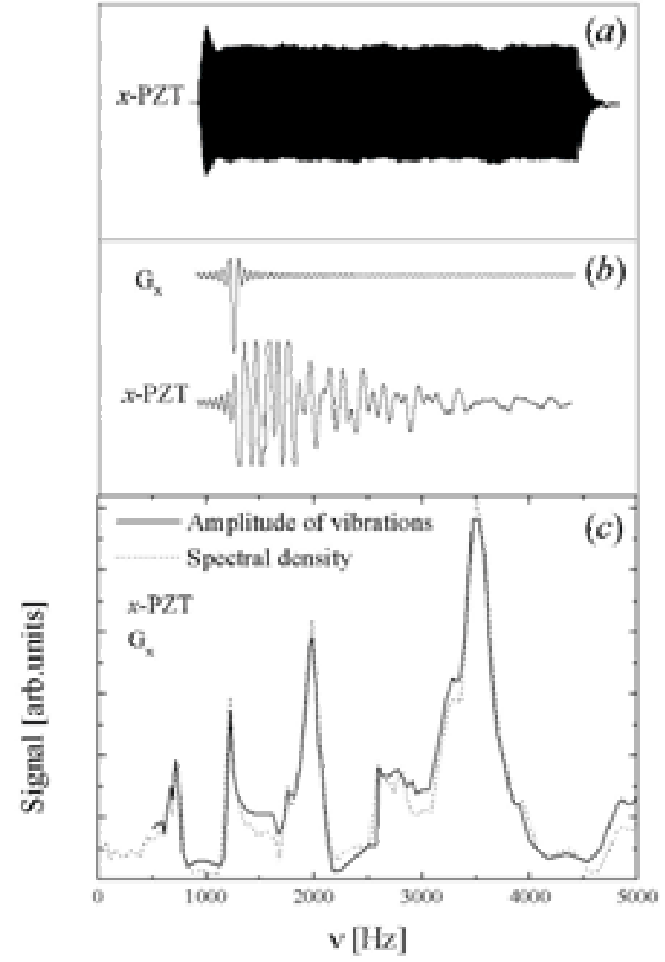

FIG. 5: (a) $x$-PZT signal caused by a 500-period sine-shaped pulse in the $G_{x}$-gradient coil. Gradient amplitude $=2.5 \mathrm{mT} / \mathrm{m}$, pulse duration $=400 \mathrm{~ms}(n=1250 \mathrm{~Hz})$. (b) $x$-PZT signal caused by a $300-$ period sinc-shaped pulse in the $G_{x}$-gradient coil. Maximum gradient amplitude $=10 \mathrm{mT} / \mathrm{m}$, pulse duration $=50 \mathrm{~ms}$. (c) Amplitude of vibrations in the assembly due to sine-gradient pulses (solid line) and sinc-gradient pulses (dashed line) in the $G_{x}$ coil, as a function of frequency.

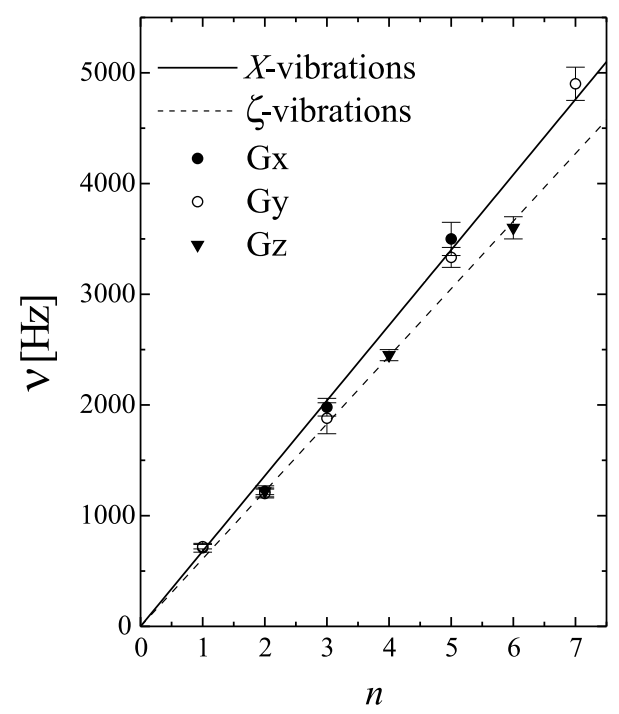

FIG. 6: Frequency of resonant modes caused by sinc-shaped pulses in the $G_{x^{-}}, G_{y^{-}}$and $G_{z}$-gradient coils as a function of $n$. Solid and dashed lines are linear fits that represent Eqs. (8) and (15), for $k<<$ $\omega_{n}$, and $K<<\Omega_{n}$.
$G_{z}$-spectrum peaks at different frequencies than $G_{x^{-}}$and $G_{y^{-}}$ spectra. This is due to the underlying difference in the current distributions and in the velocity of traveling waves for $\zeta$ - and $X$-vibrations. Unfortunately, we were unable to make a comparison between theory and experiment for the amplitudes of vibration, because for this particular gradient coil set the current distributions were unavailable to us. Such an analysis would be further complicated by the non-uniform frequency response of PZT microphones. Using the results of the impulse response experiment and Eqs. (8) and (15), high-order resonant modes were identified. Fig. 6 plots, for each gradient, the resonance frequency as a function of $n$. Transverse gradients produce odd normal modes of axis-vibration, while the longitudinal gradient produces even normal modes of surfacevibration, in agreement with the string model. Nevertheless, the $n=2$ resonant mode of surface-vibration is also produced by transverse gradients, probably due to unbalanced fixations of coil ends. The slope of these data was used to determine the velocity of traveling waves to be $c=1500$ for $\zeta$-vibrations and $C=1733 \mathrm{~m} / \mathrm{s}$ for $X$-vibrations.

\section{CONCLUSION}

Acoustic noise in MRI scanners is a consequence of the vibrations of the gradient coil assembly. We developed a simple model for the gradient coil vibrations, in which the coil assembly is represented by an elastic string that is acted upon by a distributed Lorentz force. The string model makes a few simple predictions regarding coil vibrations. First, the amplitude of vibrations is predicted to be proportional to the magnetic field strength, in agreement with previous measurements. Second, lighter coils or coils with larger radius should present large vibrations amplitudes. Furthermore, the string model predicts that the frequencies of normal vibration modes are determined by the coil length and the elastic properties of the materials in the assembly. The decay of vibrations after a gradient pulse is a consequence of dissipative processes that depend on the viscosity of the materials in the assembly. As a result, the width of resonance modes increases linearly with the viscosity of the assembly. Finally, as a consequence of the symmetry of current distributions, it is predicted that longitudinal coils produce anti-symmetrical modes of vibrations, while transverse coils produce symmetrical modes of vibrations. Again, these theoretical predictions are in general agreement with experimentally observed vibration modes. Ultimately, the knowledge gained from this study should be helpful in reducing acoustic noise in MRI scanners. 
[1] S. A. Counter, A. Olofsson, H. F. Grahn, and E. Borg, J. Magn. Reson. Imaging 7, 606 (1997).

[2] P. A. Bandettini, A. Jesmanowicz, J. Van Kylen, R. M. Birn, and J. S. Hyde, Magn. Reson. Med. 39, 410 (1998).

[3] G. F. Eden, J. E. Joseph, H. E. Brown, C. P. Brown, and T. A. Zeffiro, Magn. Reson. Med. 41, 13 (1999).

[4] Z-H Cho, S-C Chung, D-W Lim, E. K. Wong, Magn. Reson. Med. 39, 331 (1998).

[5] M. R. Elliott, R. W. Bowtell, and P. G. Morris, Magn. Reson. Med. 41, 1230 (1999).

[6] Y. Yang, A. Engelien, W. Engelien, S. Xu, E. Stern, and D. A. Silbersweig, Magn. Reson. Med 43, 185 (2000).

[7] J. Hennig, and M. Hodapp, BURST imaging. MAGMA 1, 39 (1993).

[8] F. Hennel, F. Girard, and T. Loenneker, Magn. Reson. Med. 42, 6 (1999).

[9] F. Hennel J. Magn. Reson. Imaging 13, 960 (2001).

[10] Z. H. Cho, S. T. Chung, J. Y. Chung, S. H. Park, J. S. Kim, C.
H. Moon, and I. K. Hong, Magn. Reson. Med. 39, 317 (1998). [11] P. Mansfield, B. L. W. Chapman, R. Bowtell, P. Glover, R. Coxon, and P. R. Harvey, Magn. Reson. Med. 33, 276 (1995).

[12] P. Mansfield, and B. Haywood MAGMA 8 (Suppl), 55 (1999).

[13] P. Mansfield, B. Haywood, and R. Coxon, Magn. Reson. Med. 46, 807 (2001)

[14] D. L. Price, J. P. De Wilde, A. M. Papadaki, J. S. Curran, and R. I. Kitney J. Magn. Reson. Imaging 13, 288 (2001).

[15] Y. Wu, B. A. Chronik, C. Bowen, C. K. Mechefske, and B. K. Rutt, Magn. Reson. Med. 44, 532 (2000).

[16] R. A. Hedeen, and W. A. Edelstein, Magn. Reson. Med. 37, 7 (1997).

[17] P. Mansfield, P. M. Glover, J. Beaumont, Magn. Reson. Med. 39, 539 (1998).

[18] D. Tomasi, Magn. Reson. Med. 45, 505 (2001).

[19] D. Tomasi, and T. Ernst J. Magn. Reson. Imaging 18, 128 (2003). 